\title{
Induction of Amodiaquine Metabolism by Rifampicin Following Concurrent Administration in Healthy Volunteers
}

\author{
Adebusuyi Akande Ademisoye ${ }^{1}$, Julius Olugbenga Soyinka ${ }^{1 *}$, \\ Samuel Olanrewaju Olawoye ${ }^{1}$, Sharon Iyobor Igbinoba ${ }^{2}$, \\ Samuel Anu Olowookere ${ }^{3}$, Adelola Taiwo Ademisoye ${ }^{1}$ and Cyprian Ogbona Onyeji ${ }^{1}$ \\ ${ }^{1}$ Department of Pharmaceutical Chemistry, Obafemi Awolowo University, Ile-Ife, Nigeria; ${ }^{2}$ Department of Clinical Pharmacy and \\ Pharmacy Administration, Obafemi Awolowo University, Ile-Ife, Nigeria; ${ }^{3}$ Department of Community Health, College of Health Sciences, \\ Obafemi Awolowo University, Ile-Ife, Nigeria
}

\begin{abstract}
Background and objective: Malaria and tuberculosis remain endemic in tropical regions and most often coexist, increasing the burden of malaria mortality due to unintended interactions of the co-administered drugs employed in the management of the infections. Rifampicin (RIF) and amodiaquine (ADQ) are likely to be administered concurrently in the treatment of patients with tuberculosis and malaria. The metabolism of ADQ is mediated principally by CYP2C8, while RIF is a known inducer of this enzyme. This study, therefore, investigated the effect of RIF on the disposition of $A D Q$, a major partner drug in the first-line treatment against uncomplicated malaria in line with the World Health Organization recommendations.
\end{abstract}

Methods: Sixteen healthy volunteers received oral $150 \mathrm{mg}$ dose of RIF daily for 5 days with or without oral 600 $\mathrm{mg}$ single dose of $\mathrm{ADQ}$ on the fifth day ( $5^{\text {th }}$ dose) with a 4-week wash out period, in a crossover fashion. Blood samples were collected at predetermined time intervals of $0,1,2,4,6,8,12,24,36$ and $48 \mathrm{~h}$. Plasma samples were analyzed for ADQ and its major metabolite desethylamodiaquine using a validated RP-high-performance liquid chromatography. Pharmacokinetic parameters were obtained using appropriate software and subjected to appropriate statistical analysis.

Results: Coadministration of $A D Q$ and RIF resulted in significant decreases in the critical pharmacokinetic parameters of $A D Q$, such as area under the curve $\left(A \cup C_{0-\infty}\right)$ of about $66 \%$, time to peak plasma concentration (Tmax) of about $10 \%$, maximum plasma concentration of about $44 \%$, and elimination half-life of about $55 \%$, while the $A \mathrm{CC}_{0-\infty}$ and Tmax of the main metabolite desethylamodiaquine increased about 2-fold and 3-fold respectively during the coadministration of RIF with ADQ. The metabolic ratio increased significantly, from 1.55 to 2.68 . The $A \cup C_{0-\infty}$ and Tmax of the drug $A D Q$, as well as the maximum concentration of both the drug and its metabolite fell outside the point of estimates of the test/reference ratio of the geometric means of $80-125 \%$ of bioequivalence range.

Conclusions: An interaction was established during coadministration of RIF and ADQ, confirming RIF as a strong inducer of CYP2C8 in vivo, which may lead to malaria therapeutic failure or adverse drug reactions with ADQ

Keywords: Amodiaquine; CYP2C8; Desethylamodiaquine; Induction; Rifampicin. Abbreviations: $\mathrm{AUC}_{\mathrm{T}}$, area under plasma concentration-time curve; $\mathrm{BE}$, bioequivalence; CL/F, oral clearance; Cmax, maximum concentration; CYP, cytochrome P450; DEAQ, desethylamodiaquine; HPLC, high-performance liquid chromatography; RIF, rifampicin; $\mathrm{T}^{1} \mathrm{2}$, elimination half-life; $\mathrm{T}^{1} \mathrm{2} \mathrm{b}$, terminal elimination half-life; $\mathrm{TB}$, tuberculosis; Tmax, time to reach maximum concentration.

Received: November 21, 2017; Revised: March 23, 2018; Accepted: March 27, 2018 *Correspondence to: Julius O. Soyinka, Department of Pharmaceutical Chemistry, Faculty of Pharmacy, Obafemi Awolowo University, Ile-Ife, Nigeria. Tel: +2348035822785; E-mail: juliussoyinka@gmail.com

How to cite this article: Ademisoye AA, Soyinka JO, Olawoye SO, Igbinoba SI, Olowookere SA, Ademisoye AT, Onyeji CO. Induction of Amodiaquine Metabolism by Rifampicin Following Concurrent Administration in Healthy Volunteers. J Explor Res Pharmacol 2018;3(3):71-77. doi: 10.14218/JERP.2017.00024. and contribute to the rate at which resistance to ADQ develops.

\section{Introduction}

Between 2010 and 2015, concerted efforts aimed at controlling malaria infection globally reduced the incidence of malaria by $21 \%$, its mortality rates by $29 \%$ among at-risk populations and by $35 \%$ among children under 5 years of age. ${ }^{1}$ In spite of these statistics, 
malaria remains one of the most serious health problems worldwide. ${ }^{2}$ Plasmodium falciparum, which causes the most severe form of malaria, is predominant in Africa; and, in 2015, about 212 million cases of malaria occurred worldwide, mostly in Africans (90\%). ${ }^{3}$ Incidentally, in regions with high incidence of malaria infections, such as sub-Saharan Africa, malaria may coexist with other infections. ${ }^{4}$

Tuberculosis (TB), an infection caused by Mycobacterium tuberculosis, is one of the top 10 leading tropical diseases that require long-term therapies. TB and malaria are the most prevalent bacterial and parasitic infections that coexist frequently in humans. ${ }^{5}$ This can potentially contribute to morbidity and mortality in impoverished populations in the tropics. ${ }^{6}$ Moreover, concurrent infections involving TB and malaria have been reported to modify immune response in the coinfection situation and to increase antibacterial intolerance, ${ }^{7}$ which may be detrimental to the prognosis of TB. ${ }^{6}$ In addition, malaria infection can result in rapid increase in parasite burden if there is a delay in or application of ineffective treatment, with reported case fatality rates of $10 \%$ to $20 \%{ }^{8}$

Therefore, a treatment goal for TB patients with malaria will be the prevention or effective treatment of malaria in TB patients in order to reduce overall morbidity and mortality. ${ }^{6,9-11}$ For instance, induction of CYP3A4-mediated quinine metabolism in a patient infected with the human immunodeficiency virus by the antiretroviral drug nevirapine worsened malaria symptoms, and the clinical deterioration was only abated when quinine was substituted with proguanil/atovaquone, a non-CYP3A4 substrate. ${ }^{12}$ Concurrent sulphamethoxazole and trimethoprim combination with amodiaquine (ADQ) has been found to increase pharmacokinetic parameters of ADQ, due to inhibition of its metabolism when concurrently administered. ${ }^{13}$

ADQ is a frontline antimalarial recommended by the World Health Organization for artemisinin derivative-based combination therapy. ${ }^{14}$ It is available in tablets of $150-600 \mathrm{mg}$, and the recommended dose is $10 \mathrm{mg} / \mathrm{kg}$ of ADQ base once daily for 3 days, usually in combination with other antimalarial, such as artesunate. ADQ is almost entirely metabolized by the polymorphic cytochrome P450 (CYP) isoform 2C8 to the pharmacologically active desethylamodiaquine (DEAQ). ${ }^{15}$ Expression of each CYP is influenced by a unique combination of mechanisms and factors, including genetic polymorphisms, induction by xenobiotics, regulation by cytokines hormones and during disease states, as well as sex, age and others. ${ }^{16}$

Multiallelic genetic polymorphisms, which strongly depend on ethnicity, play a major role in the function of CYPs 2D6, 2C8, 2C9 and $2 \mathrm{~B} 6$, and lead to distinct pharmacogenetic phenotypes, termed as poor, intermediate, extensive and ultra-rapid metabolizers. ${ }^{17}$ For these CYPs, the evidence for clinical significance regarding adverse drug reactions, drug efficacy and dose requirement is rapidly growing. Knowledge of the intrinsic and extrinsic factors that influence expression and function of the responsible enzymes is, thus, a prerequisite for predicting variable pharmacokinetics and drug response.

The human CYP2C subfamily consists of the four highly homologous genes, CYP2C18-CYP2C19-CYP2C9-CYP2C8, which are localized in this order (from centromere to telomere) in a 390 $\mathrm{kb}$ gene cluster on chromosome 10q23.3.18 Although CYP2C18 mRNA is highly expressed in liver, the transcript is not efficiently translated into protein and does not make significant contributions to drug metabolism. ${ }^{18} \mathrm{CYP} 2 \mathrm{C} 9$ is the highest expressed member, being expressed at similar or even higher protein levels than CYP3A4, while CYP2C8 and CYP2C19 are expressed at about 2-fold and 10 -fold lower levels. ${ }^{18}$

Similarly, rifampicin (RIF) is an important drug in the treatment of TB and is used extensively despite its broad effects on drug-drug interactions arising from its activities on most CYP and/or P-glycoproteins in the gastrointestinal tract and liver. ${ }^{19,20}$ It is available in capsules of 150-300 mg, and the recommended dose is $10 \mathrm{mg} / \mathrm{kg}$ / day; the dose should not exceed $600 \mathrm{mg}$ /day. The drug is usually given in combination with other anti TB drugs, such as isoniazid. Concurrent infections with malaria and TB may occasion the need for ADQ and RIF coadministration, ${ }^{7,9}$ and such practice may give rise to clinically important drug-drug interactions that may compromise the therapeutic outcome. RIF and lopinavir/ritonavir, ${ }^{21,22}$ in separate studies, enhanced the therapeutic activity of ADQ in mice infected with chloroquine-resistant Plasmodium berghei.

It has been reported that withdrawal of RIF from the medication regimen of a TB patient on atenolol, amlodipine and ramipril (ACE inhibitor) in the management of essential hypertension brought the fatal consequence of hypertension. ${ }^{23}$ Since ADQ is mainly metabolized by CYP2C8 and activity of this isozyme has been demonstrated to be modulated by RIF, ${ }^{21}$ theoretically, RIF is expected to induce the metabolism of ADQ. The extent of interaction between RIF and ADQ when used concurrently in man is not known. Since effective malaria treatment is important in TB patients and RIF may reduce the overall exposure to $A D Q$, this study set out to determine how RIF influences the disposition of ADQ in man.

\section{Methods}

\section{Materials}

Amodiaquine hydrochloride dihydrate (Sigma, St. Louis, MO, USA). Desethylamodiaquine (TLC PharmaChem, Ontario, Canada). Hydroxychloroquine sulphate (AK Scientific Inc., San Francisco, CA, USA). High-performance liquid chromatography (HPLC)-grade methanol and triethylamine (Scharlau Chemie, Barcelona, Spain). Analytical grade diethyl ether (Lobal Chemie, Mumbai, India). Orthophosphoric acid (Aldrich, Hamburg, Germany). Amodiaquine tablets (Camoquine ${ }^{\circledR}$ ) (Parke-Davis, Pfizer, Detroit, MI, USA). Rifampicin (Rifabon) (Bond Pharmaceuticals, Nigeria). Hydrochloric acid (Sigma-Aldrich, UK).

\section{Instrumentation}

The HPLC system (Agilent 1200 series; Agilent Technologies, Frankfurt, Germany) consisted of a binary pump with a degasser and isocratic gradient mixer, reverse-phase C18 column (150 $\mathrm{mm} \times 4.6 \mathrm{~mm}$ i.d. $\times 5 \mu \mathrm{m})($ Agilent Eclipse Plus), rheodyne valve injector loop $(20 \mu \mathrm{L})$, variable wavelength ultraviolet detector, Chromjet data integrator and Hewlett-Packard Chemstation recorder. Other equipment included a vortex mixer (XHC, Nanjing, China), centrifuge (800-CE; Jiangsu, China), vacuum filter (Supelco, Bellefonte, PA, USA), pH meter (Hanna Instruments, Wilmington, NC, USA), and micropipettes (Microlax, Milan, Italy).

\section{Ethical clearance}

Ethical clearance for the study was obtained from the Health Research Ethics Committee of the Institute of Public Health, Obafemi Awolowo University, Ile-Ife, Nigeria (Reference Number: IPH/ OAU/12/539; $15^{\text {th }}$ March, 2016).

\section{Study population}

Healthy nonsmoking men and women who were within $20 \%$ 
of their ideal bodyweight for height and sex (Metropolitan Life Scale) were deemed eligible to participate in the study. Subjects were excluded if they met one of the following criteria: pregnancy; breastfeeding; history of hypersensitivity reactions to RIF, ADQ or similar agents (mefloquine, chloroquine, quinidine, quinine); serum creatinine $>1.5$ times the upper limit of normal; liver function test $>3$ times the upper limit of normal; or evidence by history or physical examination of gastrointestinal, psychiatric, cardiovascular or neurological disorder. Electrocardiogram findings were also recorded. Nine men and seven women, who had all been certified healthy by a doctor in the team, were recruited as well, but only fourteen volunteers completed the study. The mean \pm standard deviation age and weight of the subjects was $24.75 \pm 2.8$ years (range: $18-43$ ) and $57.88 \pm 4.5 \mathrm{~kg}$ (range: 40-80) respectively. None of them had received any other drugs for at least 1 month before the study. All subjects gave written informed consent before participation in the study.

\section{Study design and drug administration}

The study was an open-label, randomized, two-period crossover pharmacokinetic study. The subjects were randomized into two groups (Groups A and B). After an overnight fast, Group A subjects received a single oral $600 \mathrm{mg}$ dose of $\mathrm{ADQ}$, while Group B received oral $150 \mathrm{mg}$ dose of RIF daily for 5 days and $600 \mathrm{mg}$ dose of ADQ with the fifth RIF dose. After allowing 4-weeks wash-out period, Group A subjects received the same drug treatment that had been given earlier to group B, and vice versa. No other drugs or alcohol were permitted during the study.

\section{Sample collection}

Before drug administration, venous blood samples $(5 \mathrm{~mL})$ were collected from all subjects for determination of serum chemistry and hematological screening. Thereafter, blood samples $(5 \mathrm{~mL})$ were drawn by venipuncture into heparinized tubes from the forearm of each subject before ADQ administration and at 1, 2, 4, 6, 8, $12,24,36$ and $48 \mathrm{~h}$ afterwards. The samples were centrifuged immediately $(3,000 \times g$ for $15 \mathrm{~m})$ to separate the plasma. The plasma aliquots were stored at $-20{ }^{\circ} \mathrm{C}$ until analysis.

\section{Drug analysis}

Plasma samples were analyzed for ADQ and DEAQ using the HPLC method reported by Adedeji et al. ${ }^{24}$ Sample extraction involved precipitation of the plasma proteins, followed by extraction as follows: in a $15 \mathrm{~mL}$ extraction tube, $1 \mathrm{~mL}$ of plasma, $1 \mathrm{~mL}$ of acetonitrile and $2 \mu \mathrm{L}$ of $50 \mu \mathrm{g} / \mathrm{mL}$ of hydroxychloroquine (internal standard) were added and centrifuged for $20 \mathrm{~min}$ at 3,000 $\times \mathrm{g}$ to precipitate the plasma protein. The supernatant was then separated into clean and dry extraction tubes, to which $2 \mathrm{~mL}$ of diethyl ether was added. The content was vortexed for $2 \mathrm{~m}$ and centrifuged for $20 \mathrm{~m}$ at $3,000 \times g$. The organic layer was transferred into taper-end tubes. The exercise was repeated with another $2 \mathrm{~mL}$ diethyl ether and the combined organic layers in the tubes were evaporated under stream of warm air. The residue was reconstituted with $1 \mathrm{~mL}$ of $0.1 \mathrm{M}$ hydrochloric acid.

A $20 \mu \mathrm{L}$ aliquot was injected onto the HPLC column by using an autosampler. The calibration procedures were as reported. ${ }^{24} \mathrm{~A}$ calibration curve, based on peak area ratio, was prepared by spiking drug-free plasma with standard solutions of ADQ and DEAQ to give concentration ranges of $100-1,000 \mathrm{ng} / \mathrm{mL}$ for both ADQ and DEAQ. The samples were taken through the extraction procedure described above. The mobile phase, consisting of $2 \%$ TEA buffer solution ( $\mathrm{pH} 2.2)$ : methanol (80:20), was prepared and pumped through the column at $1.0 \mathrm{~mL} / \mathrm{min}$. The column was set at $30^{\circ} \mathrm{C}$, while the column effluent was monitored with a variable wavelength ultraviolet detector set at $340 \mathrm{~nm}$.

\section{Data and statistical analyses}

The peak plasma concentrations (Cmax) and the time to reach peak concentration (Tmax) were noted directly from the concentrationtime profiles. Other pharmacokinetic parameters, such as terminal elimination half-life $\left(\mathrm{T}^{1} / 2 \mathrm{~b}\right)$ and oral clearance $(\mathrm{CL} / \mathrm{F})$ were calculated from individual plasma concentration-time profiles, using standard noncompartmental methods. ${ }^{25}$ For example, the total area under the plasma concentration-time curve $\left(\mathrm{AUC}_{\mathrm{T}}\right)$ was determined using the linear trapezoidal rule to the last datum and extrapolation to infinity. The area from the last datum points $(\mathrm{Ct})$ to infinity was obtained as $\mathrm{Ct} / \mathrm{b}$. The elimination rate constant, $\mathrm{b}$, was calculated by linear regression analysis of the terminal phase of the $\log$ concentration-time profile. $\mathrm{T}^{1} / 2 \mathrm{~b}$ was calculated from $0.693 / \mathrm{b}$ and $\mathrm{CL} / \mathrm{F}$ was determined from dose $/ \mathrm{AUC}_{\mathrm{T}}$. Pharmacokinetic calculations were carried out using the pharmacokinetic program WinNonlin (standard edition, version 1.5; Scientific Consultant Inc., Apex, NC, USA). In the model option for the noncompartmental analysis, the linear trapezoidal rule was used for calculation of the AUC. The Wilcoxon matched pairs signed-ranked test was used to evaluate the difference between pairs of data; a $p$-value below 0.05 was considered significant.

\section{Results}

The retention times of internal standard, DEAQ and ADQ were 3.5, 5.2 and $6.4 \mathrm{~m}$, respectively. The coefficients of variation for both the intraday and interday analyses at 150, 300 and $900 \mathrm{ng} /$ $\mathrm{mL}$ ranged from $3.10-6.86 \%$ for $\mathrm{ADQ}$, and from $4.68-8.98 \%$ for DEAQ. The mean extraction recoveries of ADQ and DEAQ were $80.0 \%$ and $78.9 \%$, respectively, at concentrations at the lower and upper limits of their calibration curves.

The mean ADQ and DEAQ plasma concentrations versus time profiles in the 14 healthy subjects who completed the study, following oral administration of single doses of ADQ $(600 \mathrm{mg})$ alone and coadministration of ADQ with RIF ( $150 \mathrm{mg}$ daily for 5 days) are illustrated in Figures 1 and 2, respectively. Tables 1 and 2 summarize the pharmacokinetic parameters (mean \pm standard deviation) for ADQ and DEAQ following a single oral administration of $600 \mathrm{mg}$ dose of ADQ to each of the subjects before and after concurrent administration of RIF (150 mg daily for 5 days), respectively. The demographic features of the study participants are presented in Table 3.

The Cmax of ADQ when administered separately was $47.43 \pm$ $6.20 \mathrm{ng} / \mathrm{mL}$, but decreased significantly to $26.49 \pm 4.39 \mathrm{ng} / \mathrm{mL}$ ( $p$ $<0.05$ ) when co-administered with RIF, resulting in about a $44 \%$ decrease, whereas the Cmax of the metabolite was $54.42 \pm 3.56$ and $59.94 \pm 3.8 \mathrm{ng} / \mathrm{mL}$ for ADQ and its concurrent administration with RIF, respectively, and was comparable to the baseline ( $p>$ $0.05)$. The Tmax was $3.43 \pm 0.98$ and $2.75 \pm 0.97 \mathrm{~h}(p>0.05)$ for ADQ alone and coadministration with RIF, respectively, but there was a 3-fold increase in the Tmax of the metabolite after single 600 $\mathrm{mg}$ dose of ADQ and its coadministration with RIF (150 mg daily 
J Explor Res Pharmacol

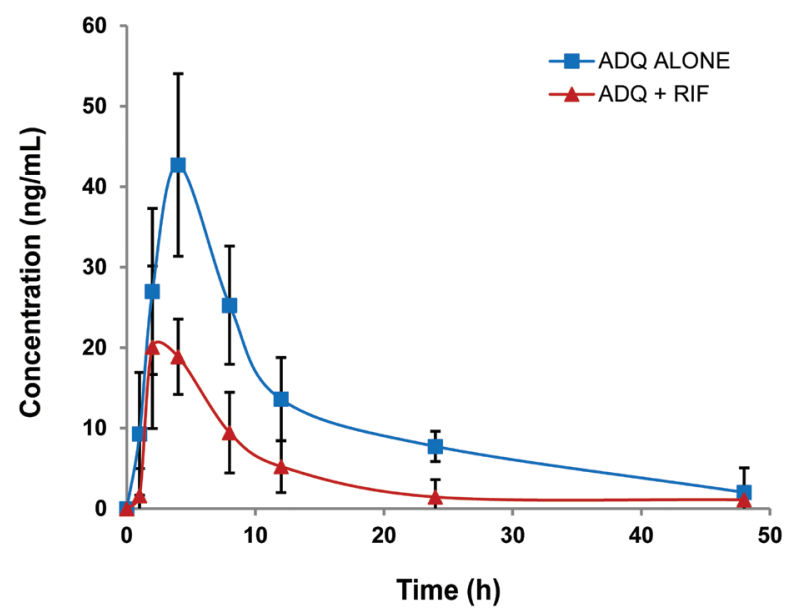

Fig. 1. Mean $A D Q$ plasma concentrations versus time profiles in 14 healthy subjects following oral administration of single dose of ADQ at $600 \mathrm{mg}$ alone and coadministration of ADQ with RIF at $150 \mathrm{mg}$ daily for 5 days. Abbreviations: ADQ, amodiaquine; RIF, rifampicin.

for 5 days), specifically $2.01 \pm 0.05$ and $6.01 \pm 1.2$ ). The Cmax of both the drug and its metabolite fell outside the bioequivalence range of $80-125 \%$, being $-58.2(49.2,68.1)$ and $9.7(6.1,13.6)$, respectively.

The Tmax of the drug also fell outside the range, being 19.1 $(9.2,30.4)$, whereas the Tmax of the metabolite was bioequivalent. There was an almost $66 \%$ fall in ADQ exposure, as depicted by the $\mathrm{AUC}_{0-\infty}$ after single dose of ADQ, which was $572.53 \pm 121.96$ $\mathrm{ng} / \mathrm{L} * \mathrm{~h}$ and $196.42 \pm 77.39 \mathrm{ng} / \mathrm{L} * \mathrm{~h}(p<0.05)$ when it was coadministered with RIF, with corresponding increase by about $90 \%$ of the $\mathrm{AUC}_{0-\infty}$ of the metabolite DEAQ $(795.53 \pm 193.84$ vs. 1,510 $\pm 942.60, p<0.05)$ during single oral dose of ADQ and concurrent administration with RIF. Only the AUC of the metabolite fell outside the $90 \%$ confidence interval range of 64.1 (43.6, 77.2), whereas the AUC for the drug was bioequivalent, being 107 (94.1, 120.5).

There was a $52 \%$ decrease in the mean residence time of ADQ when taken alone and when RIF was coadministered, with results being $17.92 \pm 10.16 \mathrm{~h}$ and $8.52 \pm 5.98 \mathrm{~h}$, respectively $(p<0.05)$. There was equally a $55 \%$ decrease in the elimination half-life $\left(\mathrm{T}_{1 / 2}\right)$ of ADQ when it was administered alone and when coadministered with RIF, being $12.35 \pm 7.27 \mathrm{~h}$ and $5.5 \pm 5.85 \mathrm{~h}$, respectively, with a corresponding significant elevation of its CL/F, from 1,087 \pm
Ademisoye AA. et al: Induction of amodiaquine by rifampicin

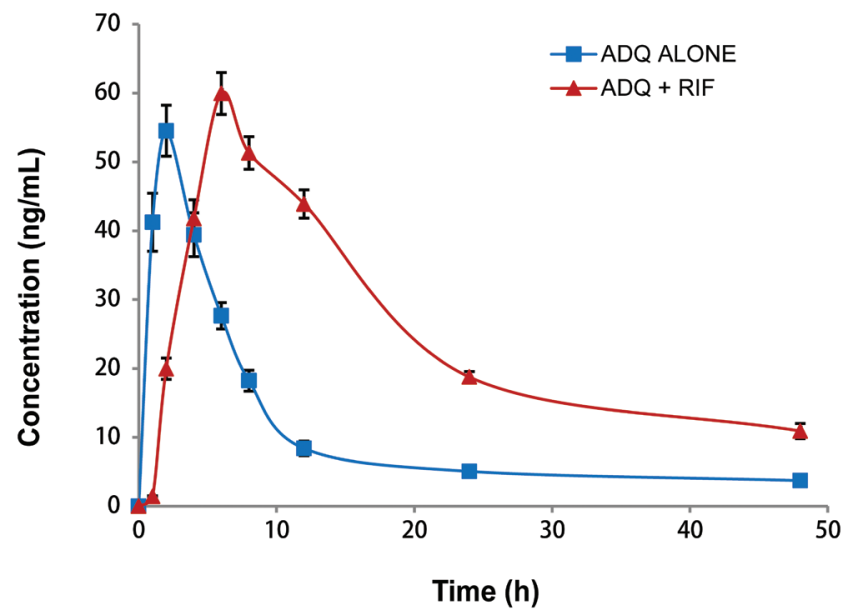

Fig. 2. Mean DEAQ concentrations versus time profile in 14 healthy subjects following oral administration of single dose of $A D Q$ at $600 \mathrm{mg}$ alone and coadministration of ADQ with RIF at $\mathbf{1 5 0} \mathrm{mg}$ daily for 5 days. Abbreviations: $A D Q$, amodiaquine; $D E A Q$, desethylamodiaquine; RIF, rifampicin.

$205.4 \mathrm{~L} / \mathrm{h}$ to $3,183 \pm 588.48 \mathrm{~L} / \mathrm{h}(p<0.05)$. The oral clearance of the metabolite decreased by about $44 \%$, from $778.18 \pm 172.0 \mathrm{~L} / \mathrm{h}$ to $436.78 \pm 125.17 \mathrm{~L} / \mathrm{h}(p<0.05)$.

The metabolic ratio, which is the AUC(metabolite)/AUC(drug), that is, the ratio of AUC of the metabolite to that of the unchanged drug, and is a known measure of extent of metabolism of a drug, was found to increase significantly, from $1.55 \pm 0.53$ to $2.68 \pm 1.85$ before and after RIF coadministration, respectively $(p<0.05)$.

\section{Discussion}

This study was designed to evaluate the plausible interaction between ADQ and RIF. Only 2 out of the 16 recruited volunteers, a male and a female, withdrew from the study after complaining about dizziness and pain at the site of blood collection, so that a total of 14 volunteers completed the study. Their withdrawal, however, did not in any way affect the conduct, the goal nor the findings of the study.

There is a constant stream of new information about drug interactions in patients with malarial infection, who may also need to take antimalarial drugs together with drugs for other ailments.

Table 1. Derived pharmacokinetic parameters of ADQ following oral administration of single $600 \mathrm{mg}$ dose of the drug to each of 14 volunteers and when coadministered with multiple doses of RIF at $150 \mathrm{mg}$ daily for 5 days

\begin{tabular}{|c|c|c|c|}
\hline PK parameters & ADQ alone & ADQ+RIF & Statistics with $90 \% \mathrm{Cl}$ \\
\hline Cmax, ng/mL & $47.43 \pm 6.20$ & $26.49 \pm 4.40$ & $p<0.05-58.2(49.2,68.1)^{*}$ \\
\hline Tmax, h & $3.43 \pm 0.98$ & $2.75 \pm 0.97$ & $p>0.0519 .1(9.2,30.4)^{*}$ \\
\hline $\mathrm{AUC}_{0-\infty}, \mathrm{ng} / \mathrm{mL}^{*} \mathrm{~h}$ & $575.53 \pm 121.96$ & $196.42 \pm 77.39$ & $p<0.05107(94.1,120.5)$ \\
\hline $\mathrm{T} 1 / 2, \mathrm{~h}$ & $12.35 \pm 7.27$ & $5.60 \pm 5.85$ & $p<0.05$ \\
\hline MRT, h & $17.92 \pm 10.16$ & $8.52 \pm 5.98$ & $p<0.05$ \\
\hline $\mathrm{CL} / \mathrm{F}, \mathrm{L} / \mathrm{h}$ & $1,087 \pm 205.4$ & $3,183 \pm 588.48$ & $p<0.05$ \\
\hline$V d / f, L$ & $73.04 \pm 27.96$ & $473.7 \pm 177.28$ & $p<0.05$ \\
\hline
\end{tabular}

*Values within the $80-125 \%$ bioequivalence range. Abbreviations: $\mathrm{ADQ}$, amodiaquine; $\mathrm{Cl}$, confidence interval; $\mathrm{CL} / \mathrm{F}$, oral clearance; $\mathrm{MRT}$, mean residence time; $\mathrm{PK}$, pharmacokinetic; RIF, rifampicin. 
Table 2. Derived pharmacokinetic parameters of desethylamodiaquine following oral administration of single $600 \mathrm{mg}$ dose of $A D Q$ to each of 14 volunteers and when coadministered with multiple doses of RIF at $\mathbf{1 5 0} \mathrm{mg}$ daily for $\mathbf{5}$ days

\begin{tabular}{llll}
\hline PK parameters & ADQ alone & ADQ+RIF & Statistics with $90 \%$ Cl \\
\hline Cmax, ng/mL & $54.42 \pm 3.56$ & $59.94 \pm 2.38$ & $p>0.059 .7(6.1,13.6)^{*}$ \\
Tmax, h & $2.01 \pm 0.05$ & $6.01 \pm 1.20$ & $p<0.05111 .0(99,123.1)$ \\
AUC $_{0-\infty}, \mathrm{ng} / \mathrm{mL}^{*} \mathrm{~h}$ & $795.53 \pm 193.84$ & $1,510.63 \pm 942.60$ & $p<0.0564 .1(43.6,77.2)^{*}$ \\
Metabolic ratio $_{\mathrm{MRT}, \mathrm{h}}$ & $1.55 \pm 0.53$ & $2.68 \pm 1.85$ & $p<0.05$ \\
$\mathrm{CL} / \mathrm{F}, \mathrm{L} / \mathrm{h}$ & $6.30 \pm 1.12$ & $12.55 \pm 1.03$ & $p<0.05$ \\
$\mathrm{Vd} / \mathrm{f}, \mathrm{L}$ & $778.18 \pm 172.0$ & $436.78 \pm 125.17$ & $p<0.05$ \\
\hline
\end{tabular}

*Values within the $80-125 \%$ bioequivalence range. Abbreviations: $\mathrm{ADQ}$, amodiaquine; $\mathrm{Cl}$, confidence interval; $\mathrm{CL} / \mathrm{F}$, oral clearance; $\mathrm{MRT}$, mean residence time; $\mathrm{PK}$, pharmacokinetic; RIF, rifampicin.

RIF has been shown to have the potential to interact with a range of other drugs, ${ }^{20,21,23}$ and healthcare providers need to be aware of the basis of these interactions and the potential impact on therapy. Clinical situations that require antibiotic therapy are prevalent in many parts of the world, including sub-Saharan Africa, where malaria is endemic. ${ }^{26}$ Moreover, the comorbidities increase the potential for drug interactions during poly therapeutics.

Information on interactions between antimalarial and antibiotics are limited. Combining antibiotic medications with an antimalarial can result in treatment failure when plasma concentrations fall below therapeutic levels or toxicity resulting from increased plasma drug levels. Our study evaluated the possible interaction between ADQ and RIF, when coadministered in healthy human subjects. The results from the present study indicated that ADQ was rapidly absorbed after oral administration in all the subjects. The Tmaxs of ADQ were $2.0 \pm 0.05$ and $6.01 \pm 1.2 \mathrm{~h}$ for single dose ADQ and coadministration with RIF, respectively, which revealed enhanced exposure of the drug to the body, with corresponding values for the metabolite DEAQ of $3.43 \pm 0.98$ versus $2.75 \pm 0.97$, respectively.

While the Tmax for the metabolite did not translate to clinical relevance, as assessed by $90 \%$ confidence intervals for its mean ratios that are bioequivalent, the Tmax of the unchanged drug had value of $19.1(9.2,30.4)$ that are not within the range of $80-125 \%$. The point estimates of the test/reference ratio of the geometric means for the Cmax of the unchanged drug and its metabolite were $58.2(49.2,68.1)$ and $9.7(6.1,13.6)$, respectively, falling outside the bioequivalence range. Although ADQ is not known to have a

Table 3. Demographic features of the study participants

\begin{tabular}{ll}
\hline Demographic features & \\
\hline Participants, total & 16 \\
Participants, completed the study & 14 \\
Sex, female/male & $6 / 8$ \\
\hline & Mean \pm SD $^{\mathrm{a}}$ \\
\hline Age, years & $24.75 \pm 2.80$ \\
Weight, kg & $57.88 \pm 4.50$ \\
Height, $\mathrm{m}$ & $1.57 \pm 0.12$ \\
BMI, $\mathrm{kg} / \mathrm{m}^{2}$ & $24.80 \pm 4.20$ \\
\hline
\end{tabular}

aMean \pm the standard deviation of demographic features. Abbreviations: BMI, body mass index; SD, standard deviation. narrow therapeutic window, the significant elevation of the Cmax may explain some of the observed adverse reactions, such as agranulocytosis and hepatitis. ${ }^{27}$

Drug toxicities must be acceptable and cause less harm than the malaria itself, in order to encourage compliance and reduce mortality. ${ }^{28}$ The $\mathrm{AUC}_{0-\alpha}$ after single dose of ADQ alone was $572.53 \pm$ $121.9644 \mathrm{ng} / \mathrm{L} * \mathrm{~h}$, while the value of $196.42 \pm 77.39 \mathrm{ng} / \mathrm{L} * \mathrm{~h}(66 \%$ decrease in exposure to ADQ) was obtained when it was coadministered with RIF. Also, the $\mathrm{AUC}_{0-\infty}$ values of DEAQ were 795.53 \pm 193.84 and $1,510.63 \pm 942.60 \mathrm{ng} / \mathrm{mL}^{*} \mathrm{~h}$ (representing $90 \%$ increase in values) for ADQ and pretreatment with RIF. The apparent $\mathrm{CL} / \mathrm{F}$ for ADQ was $1,087 \pm 205.4 \mathrm{~L} / \mathrm{h}$ and $3,183 \pm 588.48 \mathrm{~L} / \mathrm{h}$ (about $200 \%$ increase), while the DEAQ clearance was $778.18 \pm$ $172 \mathrm{~L} / \mathrm{h}$ and $436.78 \pm 125.17 \mathrm{~L} / \mathrm{h}$ (about $44 \%$ decrease). The apparent fall in the exposure to ADQ could be a result of significant oral clearance, amounting to about $200 \%$ when ADQ was coadministered with RIF.

RIF is a major player in induction of many enzymes, including CYP2C $8,{ }^{20,21}$ a notable enzyme in the metabolism of ADQ, ${ }^{14,22}$ which may have clinical significance during antimalarial therapy. Therefore, the observed decrease in plasma ADQ exposure found in this study, seen as significant decreases in Cmax, $\mathrm{T}^{1 / 2}$ and $\mathrm{AUC}_{\mathrm{T}}$ of the drug following concurrent administration of RIF, is most probably attributable to induction of CYP2C 8 by RIF, the enzyme that catalyzes formation of DEAQ. This assertion is supported by the observed corresponding increase in the Cmax and AUC of the metabolite.

The stimulatory effects on the metabolism of ADQ can allow the body to detoxify as the plasma concentration falls below therapeutic levels, conferring advantage of the enhanced drug's action through increased activity of the metabolite DEAQ. For instance, in a 3-day dosage, blood concentrations higher than breakpoints of $135 \mathrm{ng} / \mathrm{mL}$ was associated with treatment success in the management of uncomplicated Plasmodium falciparum. ${ }^{29}$ In addition, the extra-hepatic metabolism by CYP1A1 and 1B1 reported by some researchers may constitute less significance in the overall metabolism of ADQ, which may have contributed to the fall. ${ }^{30}$

The $90 \%$ confidence intervals computed for the ratios of the geometric means for ADQ and the metabolite clearly showed that there was interaction for the point of estimates for $\mathrm{AUC}_{0-\infty}$ of the metabolite which fell outside the $80-125 \%$ range for ADQ and coadministration with RIF. AUC is known to be directly proportional to dose and may be disproportional if there are saturable pathways for some routes of elimination. The enhanced AUC of the metabolite may increase as dosage increases and will worsen if coadministered with inducers such as RIF, due to formation of the 
toxic metabolite amodiaquine quinoneimine, known for causing adverse effects of ADQ. ${ }^{31,32}$

The mean residence time of ADQ also showed a significant decrease in values by about $52 \%$ when taken alone and when RIF was coadministered with it $(17.92 \pm 10.16$ vs. $8.52 \pm 5.98$ h). Similarly, the $\mathrm{T}_{1 / 2}$ for ADQ showed a significant decrease in the presence of RIF, resulting in more than $50 \%$ shortening of the half-life (12.35 $\pm 7.27 \mathrm{~h} v$ s. $5.5 \pm 5.85 \mathrm{~h}$ ) with wide interindividual variation, as observed in the deviations from the mean values. It is known that ADQ exhibits interindividual variations in its pharmacokinetics. ${ }^{33}$ This is especially true because genetic polymorphisms can characterize CYP2C8 in the metabolism of drugs and provide basis for pharmacokinetic variations in individuals as well as responses to drug actions. ${ }^{34}$

There was occurrence of a pronounced increase in the metabolic ratio. This parameter, AUC (metabolite)/AUC (drug), the ratio of AUC of the metabolite to that of the unchanged drug, is a known measure of the extent of metabolism of a drug. This was found to increase from $1.55 \pm 0.53$ to $2.68 \pm 1.85$ in the absence and presence of RIF, respectively. The marked increase in the metabolic ratio of ADQ further strengthens the point that a metabolic interaction occurs between ADQ and RIF, and the hypothesis that RIF induces the metabolism of ADQ.

Since antimalarial activity is partly dependent on the metabolite DEAQ, it is evident that ADQ had been rapidly metabolized to its metabolite and this can enhance the synergistic activity of ADQ and DEAQ. On the other hand, with the increased levels of the metabolite, there is a higher possibility of the formation of the toxic ADQ metabolite quinoneimine. This toxic metabolite is a product of conversion from DEAQ by enzymes other than CYP2C8 and is reported to be responsible for some of the reported adverse drug effects of ADQ. ${ }^{31,32}$

The question remains whether the efficacy of ADQ is affected by a gene polymorphism. Genotype-inferred low metabolizers are found in $1-4 \%$ of African populations, which corresponds to millions of expected exposures to the drug. ${ }^{35}$ It is necessary to investigate the possible relevance, or lack of relevance, of CYP2C8 polymorphisms in the present and future efficacy of ADQ. To date, several nucleotide sequence variations have been identified in CYP2C8. CYP2C8*3 (c.416G > A, p.Arg139Lys and c.1196A $>\mathrm{G}$, p.Lys399Arg) is the most frequent variant allele changing the amino acid sequence of CYP2C8 in the Caucasian population, with an allele frequency of about $10-20 \% .{ }^{36}$ On the other hand, the most common amino acid changing polymorphism in black populations is CYP2C8*2 (c.805A > T, p.Ile269Phe), but it is very rare in Caucasians. ${ }^{36}$ In vitro studies have suggested that CYP2C $8 * 3$ has reduced activity for metabolizing paclitaxel and ADQ, ${ }^{37,38}$ but amiodarone $\mathrm{N}$-demethylation was not affected by this polymorphism. ${ }^{39}$ It is therefore necessary to evaluate the in vivo effect of $\mathrm{CYP} 2 \mathrm{C} 8 * 3$ on the pharmacokinetics of ADQ.

\section{Future research directions}

The clinical significance of this study would have been enhanced by also investigating the effect of ADQ coadministration on the disposition kinetics of RIF, and by carrying out the bidirectional pharmacokinetic interaction studies on patients with malaria. This is because there is a possibility of changes occurring in the pharmacokinetic profiles of these drugs due to malaria infection. More studies are needed to further evaluate the impact of this interaction and of CYP2C8 genetic polymorphisms on the clinical outcomes of treatment.

\section{Conclusions}

This study evaluated, for the first time, the effect of RIF coadministration on the pharmacokinetics of ADQ in healthy adult volunteers. The study demonstrated that concurrent administration of RIF, a known inducer of CYP2C8, with ADQ, a substrate of the isoenzyme, results in a significant reduction in the plasma levels of the antimalarial drug. Plasma levels of DEAQ, the major metabolite of $\mathrm{ADQ}$, are elevated in the presence of RIF.

Adjustment of the ADQ dose may be necessary when the drug is coadministered with RIF, but this should be balanced against the potential increase in toxicity that may be associated with elevated plasma levels of the metabolite. There is a need to further determine the clinical implications for the extensive formation of the metabolite in this interaction, since DEAQ has potential for further metabolism to toxic metabolites when there is elevated plasma level, and this may be associated with increased toxicity.

\section{Acknowledgments}

The authors appreciate the support of Sam Ace Pharmaceutical Company Nigeria Limited, for providing the HPLC facility used in the analysis of drugs in the plasma samples of this study. The authors are also sincerely grateful to the commitment of the subjects who participated in the study and the assistance of the technical staff of the Department of Pharmaceutical Chemistry, Obafemi Awolowo University, Nigeria, in making some materials and equipment available during the research study. This work was conducted using personal funds of the researchers, and no external funding or grant was received.

\section{Conflict of interest}

The authors have no conflict of interests related to this publication.

\section{Author contributions}

Designing the research (JOS, COO, AAA, SII), recruiting volunteers (JOS, AAA, ATA, SOO, SII, SAO), certifying medical fitness of volunteers (SAO), analyzing samples (JOS, AAA, ATA, SOO, SII), preparing the manuscript (JOS, AAA, ATA, SOO, SII, SAO, $\mathrm{COO})$.

\section{References}

[1] World Health Organization WHO. Available from: http://www.who. int/mediacentre/factsheets/fs094/en/ accessed 8/04/17. Accessed 2015.

[2] World Health Organization WHO. Available from: http://www.who.int/ mediacentre/factsheets/fs104/en/ accessed 8/4/17. Accessed 2017.

[3] World Health Organization (WHO). World Malaria Report. Geneve, Switzerland. 2016.

[4] Gwer S, Newton CRJC, Berkley JA. Over-diagnosis and co-morbidity of severe malaria in African children: A guide for clinicians. Am J Trop Med Hyg 2007;77(Suppl 6):6-13.

[5] Etiaba E, Onwujekwe O, Uzochukwu B, Uguru N, Okoronkwo I, Adjagba A. What co-morbidities do people with malaria have and what are their patterns of health seeking in Nigeria? Niger J Clin Pract 2015;18(1):22-26. doi:10.4103/1119-3077.146974.

[6] Scott JA, Berkley JA, Mwangi I, Ochola L, Uyoga S, Macharia A, et 
al. Relation between falciparum malaria and bacteremia in Kenyan children: a population-based, case-control study and a longitudinal study. Lancet 2011;378(9799):1316-1323. doi:10.1016/S01406736(11)60888-X.

[7] Chapman AL, Munkanta M, Wilkinson KA, Pathan AA, Ewer K, Ayles $\mathrm{H}$, et al. Rapid detection of active and latent tuberculosis infection in HIV-positive individuals by enumeration of Mycobacterium tuberculosis-specific T cells. AIDS 2002;16(17):2285-2293.

[8] Chavale H, Santos-Oliveira JR, Da-Cruz AM, Enosse S. Enhanced $T$ cell activation in Plasmodium falciparum malaria-infected human immunodeficiency virus-1 patients from Mozambique. Mem Inst Oswaldo Cruz 2012;107(8):985-992. doi:10.1590/S007402762012000800004.

[9] Sandlund J, Naucler P, Dashti S, Shokri A, Eriksson S, Hjertqvist M, et al. Bacterial co-infections in travelers with malaria: rationale for antibiotic therapy. J Clin Microbiol 2013;51(1):15-21. doi:10.1128/ JCM.02149-12.

[10] Mueller AK, Behrends J, Blank J, Schaible UE, Schneider BE. An experimental model to study tuberculosis-malaria coinfection upon natural transmission of Mycobacterium tuberculosis and Plasmodium berghei. J Vis Exp 2014;(84):e50829. doi:10.3791/50829.

[11] Audu RA, Onwujekwe DI, Onubogu CC, Adedoyin JA, Onyejepu N, Mafe AG, et al. Possible impact of co-infections of tuberculosis and malaria on the $\mathrm{CD}^{+}$cell counts of HIV patients in Nigeria. Ann Afr Med 2005;4(1):10-13.

[12] Uriel A, Lewthwaite P. Malaria therapy in HIV: drug interactions between nevirapine and quinine. Int J STD AIDS 2011;22(12):768. doi:10.1258/ijsa.2009.009489.

[13] Ademisoye AA, Soyinka JO, Abdullahi AJ, Toyin AS, Ogbona OC. Effects of co-trimoxazole co-administration on the pharmacokinetics of amodiaquine in healthy volunteers. Int J Pharm Pharm Sci 2015;7(9):272-276.

[14] WHO. Global Plan for Artemisinin Resistance Containment (GPARC). Available from: http://www.who.int/malaria/publications/atoz/artemisinin_resistance_containment_2011.

[15] Li XQ, Björkman $\bar{A}$, Andersson TB, Ridderström M, Masimirembwa CM. Amodiaquine clearance and its metabolism to N-desethylamodiaquine is mediated by CYP2C8: a new high affinity and turnover enzyme-specific probe substrate. J Pharmacol Exp Ther 2002;300(2):399-407. doi:10.1124/jpet.300.2.399.

[16] Zanger UM, Schwab M. Cytochrome P450 enzymes in drug metabolism: Regulation of gene expression, enzyme activities, and impact of genetic variation. Pharmacol Ther 2013;138(1):103-141. doi:10.1016/j.pharmthera.2012.12.007.

[17] Leimar O. The evolution of phenotypic polymorphism: randomized strategies versus evolutionary branching. Am Nat 2005;165(6):669681. doi:10.1086/429566

[18] Goldstein JA, de Morais SM. Biochemistry and molecular biology of the human CYP2C subfamily. Pharmacogenetics 1994;4(6):285-299.

[19] Li XX, Zhou XN. Co-infection of tuberculosis and parasitic diseases in humans: a systematic review. Parasit Vectors 2013;6:79. doi:10.1186/1756-3305-6-79.

[20] Valadas E, Gomes A, Sutre A, Brilha S, Wete A, Hänscheid T, et al. Tuberculosis with malaria or HIV co-infection in a large hospital in Luanda, Angola. J Infect Dev Ctries 2013;7(3):269-272. doi:10.3855/ jidc. 2703.

[21] Badejo JA, Abiodun OO, Akinola O, Happi CT, Sowunmi A, Gbotosho GO. Interaction between rifampicin, amodiaquine and artemeter in mice infected with resistant Plasmodium beighei. Malar J 2014;13:299. doi:10.1186/1475-2875-13-299.

[22] Abiodun OO, Gbimadee N, Gbotosho GO. Lopinavir/ritonavir enhanced the antimalarial activity of amodiaquine and artesunate in a mouse model of Plasmodium berghei. J Chemother 2016;28(6):482486. doi:10.1080/1120009X.2016.1139770.

[23] Gupta N, Gupta N, Jani KK. Rifampicin (a first line antitubercular drug) is a potent liver enzyme inducer and may have drug interactions: A case report of an essential hypertension patient. Available from: https://www.bmj.com/rapid-response/2011/11/02/rifampicin-first-line-antitubercular-drug-potent-liver-enzyme-inducer-and-.

[24] Adedeji ON, Bolaji OO, Falade CO, Osonuga OA, Ademowo OG. Validation and pharmacokinetic application of a high-performance liquid chromatographic technique for determination of amodiaquine and its metabolite in plasma of patients treated with oral fixed-dose amodiaquine-artesunate combination in malaria endemic areas. Antimicrob Agents Chemother 2015;59(9):5114-5122. doi:10.1128/ AAC.04957-14.

[25] Gibaldi M, Perrier D. Pharmacokinetics. NewYork: Decker; 1982.

[26] Hopkins H, Bruxvoort KJ, Cairns ME, Chandler Cl, Leurent B, Ansah EK, et al. Impact of introduction of rapid diagnostic tests for malaria on antibiotic prescribing: analysis of observational and randomised studies in public and private healthcare settings. BMJ 2017;356:j1054. doi:10.1136/bmj.j1054.

[27] Adjei GO, Goka BQ, Rodrigues OP, Hoegberg LC, Alifrangis M, Kurtzhals J. Amodiaquine associated adverse effects after inadvertent overdose and after standard therapeutic dose. Ghana Med J 2009;43(3):135-138.

[28] Taylor WR, White NJ. Anitmalaria drug toxicity: a review. Drug Saf 2004;27(1):25-61. doi:10.2165/00002018-200427010-00003.

[29] Staedke SG, Kamya MR, Dorsey G, Gasasira A, Ndeezi G, Charlebois $E D$, et al. Amodiaquine, sulfadoxine/pyrimethamine, and combination therapy for treatment of uncomplicated falciparum malaria in Kampala, Uganda: a randomised trial. Lancet 2001;358(9279):368374. doi:10.1016/S0140-6736(01)05557-X.

[30] White NJ, Looareesuwan S, Edwards G, Phillips RE, Karbwang J, Nicholl DD, et al. Pharmacokinetics of intravenous amodiaquine. $\mathrm{Br} J$ Clin Pharmacol 1987;23(2):127-135. doi:10.1111/j.1365-2125.1987. tb03020.x.

[31] Winstanley PA, Coleman JW, Maggs JL, Breckenridge AM, Park BK. The toxicity of amodiaquine and its principal metabolites towards mononuclear leucocytes and granulocyte/monocyte colony forming units. Br J Clin Pharmacol 1990;29(4):479-485. doi:10.1111/j.1365-2125.1990.tb03667.x.

[32] Guévart E, Aguémon A. Two cases of fulminant hepatitis during a curative treatment with an artesunate-amodiaquine combination. Med Mal Infect 2009;39(1):57-60. doi:10.1016/j.medmal.2008.09.024.

[33] Li XQ, Björkman A, Andersson TB, Ridderström M, Masimirembwa CM. Amodiaquine clearance and its metabolism to N-Desethylamodiaquine is mediated by CYP2C8: a new high affinity and turnover enzyme-specific probe substrate. J Pharmacol Exp Ther 2002;300(2):399-407. doi:10.1124/jpet.300.2.399.

[34] Parikh S, Ouedraogo JB, Goldstein JA, Rosenthal PJ, Kroetz DL. Amodiaquine metabolism is impaired by common polymorphisim in $\mathrm{CY}$ P2C8: implications for malaria treatment in Africa. Clin Pharmacol Ther 2007;82(2):197-203. doi:10.1038/sj.clpt.6100122.

[35] Paganotti GM, Gramolelli S, Tabacchi F, Russo G, Modiano D, Coluzzi $\mathrm{M}$, et al. Distribution of human CYP2C8*2 allele in three different African populations. Malar J 2012;11:125. doi:10.1186/1475-287511-125.

[36] Speed WC, Kang SP, Tuck DP, Harris LN, Kidd KK. Global variation in CYP2C8-CYP2C9 functional haplotypes. Pharmacogenomics J 2009;9(4):283-290. doi:10.1038/tpj.2009.10.

[37] Lee MY, Apellániz-Ruiz M, Johansson I, Vikingsson S, Bergmann TK, Brøsen $\mathrm{K}$, et al. Role of cytochrome P450 2C8*3 (CYP2C8*3) in paclitaxel metabolism and paclitaxel-induced neurotoxicity. Pharmacogenomics 2015;16(9):929-937. doi:10.2217/pgs.15.46.

[38] Damle BD, Dowell JA, Walsky RL, Weber GL, Stogniew M, Inskeep $\mathrm{PB}$. In vitro and in vivo studies to characterize the clearance mechanism and potential cytochrome P450 interactions of anidulafungin. Antimicrob Agents Chemother 2009;53(3):1149-1156. doi:10.1128/ AAC.01279-08.

[39] Soyama A, Hanioka N, Saito Y, Murayama N, Ando M, Ozawa S, et al. Amiodarone $N$-Deethylation by $\mathrm{CYP} 2 \mathrm{C} 8$ and its Variants, CYP2C8*3 and CYP2C8 P404A. Pharmacol Toxicol 2002;91(4):174-178. doi:10.1034/j.1600-0773.2002.910404.x. 